\title{
Formulating Device-Independent Pass-by Rendezvous
}

\author{
Arisa Hayashi, Sachio Saiki, Seiki Tokunaga, Shinsuke Matsumoto and Masahide Nakamura \\ Graduate School of System Informatics, Kobe University \\ 1-1 Rokkodai, Nada, Kobe, Hyogo 657-8501, Japan \\ Email: arisa@ws.cs.kobe-u.ac.jp, masa-n@cs.kobe-u.ac.jp
}

\begin{abstract}
Developing applications with pass-by communication is usually complex and expensive, since the applications heavily depend on the hardware to detect rendezvous (i.e., proximity) of mobile entities. In order to reduce the complexity and cost of the application development, we propose a device-independent formalization of the pass-by rendezvous of mobile entities in this paper. Specifically, we first define a pass-by rendezvous as a phenomenon, where two mobile entities are closed enough at a certain time. We then derive four properties from the definition to be satisfied within any pass-by systems. The properties allow indirect pass-by rendezvous between mobile entities with different devices. Finally, we apply the proposed method to two different practical systems (Bluetooth Low Energy and GPS). In the case study, we see how the pass-by rendezvous can be implemented in each system, and see how the indirect rendezvous across the two systems can be inferred using the proposed properties.
\end{abstract}

Keywords-pass-by wireless communication, pass-by framework, M2M, mobile devices

\section{INTRODUCTION}

With the recent advancement of embedded technologies, the latest mobile devices are equipped with various sensors. IoT and M2M allow various "things" to communicate with each other. The mobile devices and the things are integrated via network to implement smarter services.

The sensors on the mobile devices realize a new type of communication, called pass-by communication [1][2]. It is a form of wireless communication triggered when a user passes by another user. When the users get close to each other, certain sensors on their handheld devices detect a rendezvous (i.e., proximity) of the devices, which triggers certain communication or actions with the mobile devices.

To make the following discussion clearer, we use the term pass-by rendezvous to refer to an event that two mobile entities get close to each other. Also, the term pass-by system is used to describe any system that detects the pass-by rendezvous with a certain technology, to implement value-added services and applications. Several pass-by systems have already appeared for smart phones [3] and handheld game consoles [4][5]. These pass-by systems are expected to create new values in various application domains.

The conventional pass-by systems, however, have limitations with respect to portability and interoperability. In general, each pass-by system detects the pass-by rendezvous using a proprietary protocol with specific sensors. Thus, applications heavily relies on the underlying hardware. Therefore, there is no compatibility between applications on different pass-by systems. An application of a pass-by system cannot be ported directly to other pass-by systems. Thus, the tight coupling between the application and the device increases development complexity and cost. Also, the lack of interoperability among different systems restricts user's choice of mobile devices. This decreases system efficiency and user's satisfaction, as well as potential services and applications, significantly.

In order to cope with the above limitations, it is essential to consider the pass-by rendezvous (as an event) and the passby system (as a device to detect the event), separately. In this paper, we therefore propose a method that formulates the passby rendezvous in a device-independent manner.

More specifically, we first define the pass-by rendezvous as a universal phenomenon where two mobile entities get close within a certain distance at a certain time. Based on the definition, we then derive four properties to be satisfied within any pass-by systems. The first two properties are symmetry and transitivity, which are derived from the nature of passby rendezvous. The last two properties are derived from spatiotemporal information of mobile entities, which allows to use geolocation systems and pass-by systems together to complement each other. The properties derive indirect pass-by rendezvous across different pass-by systems.

To see the effectiveness, we conduct a case study that applies the proposed method to two different pass-by systems. The one system is with Bluetooth Low Energy (BLE), and another is with GPS. We also show how the indirect pass-by rendezvous how the indirect rendezvous across the two systems can be inferred using the proposed properties.

\section{Formulating PASS-By REndeZVOUS}

\section{A. Definition of Pass-by Rendezvous}

To define the pass-by rendezvous without depending on any specific system or sensor, we observe a rendezvous as a

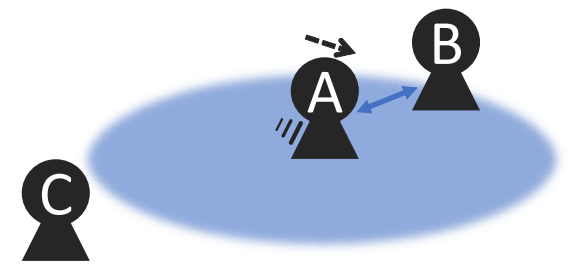

Fig. 1. Pass-by Rendezvous 
phenomenon. Figure 1 shows three mobile entities $A, B$ and $C$, where $A$ gets close to $B$, and $C$ is far from both $A$ and $B$. In this situation, we want to define a pass-by rendezvous of $A$ and $B$. Intuitively, this can be defined as a condition that "at a certain time $t, A$ and $B$ are within a close distance $l$ ".

More formally, the above condition can be defined by a function. Let $X$ be a set of all mobile entities, let $D$ be a set of all distance functions, and let $T$ be a set of all time-stamps (i.e., date time). A pass-by rendezvous is defined by a Boolean function $\chi: X \times X \times D \times T \rightarrow\{0,1\}$ such that:

$$
\chi\left(x_{1}, x_{2}, d, t\right)=\left\{\begin{array}{lll}
1 & \cdots & d\left(x_{1}, x_{2}\right)<L \text { at time } t \\
0 & \cdots & \text { otherwise }
\end{array}\right.
$$

The function returns 1 if the distance between mobile entities $x_{1}, x_{2}$ is shorter than threshold $L$ at time $t$. In Figure 1 , the shaded oval represents an area covering any rendezvous with $A$, which is determined by $L$. The arrow represents an actual distance $d(A, B)<L$. Hence, at this time $t, \chi(A, B, d, t)=1$. Note that $\chi(A, C, d, t)=0$ since $C$ is out of the area. Thus, we can define the pass-by rendezvous by $\chi$.

For convenience, we use notation $\left\langle\left\langle x_{1}, x_{2}, l, t\right\rangle\right\rangle$ to represent 4-tuples $x_{1}, x_{2}(\in X), l\left(=d\left(x_{1}, x_{2}\right)\right), t$ such that $\chi\left(x_{1}, x_{2}, d, t\right)=1$. For example, ( $\langle$ Arisa, Bob, 0.5m, 201501-23T12:34:56 $\rangle$ denotes a pass-by rendezvous, where Arisa passed by Bob at a distance of 0.5m at 12:34:56 of 2015-01-23.

\section{B. Properties of Pass-by Rendezvous}

The device-independent definition of the pass-by rendezvous yields useful properties to be satisfied within any pass-by systems.

The first property is symmetry. When $A$ gets close to $B$, we can also say that $B$ gets close to $A$. Formally, this is due to the symmetric nature of distance function.

Property P1 (Symmetry): Let $x_{1}$ and $x_{2}$ be mobile entities, and let $l=d\left(x_{1}, x_{2}\right)$, and let $t$ be a time-stamp. Then,

$$
\left\langle\left\langle x_{1}, x_{2}, l, t\right\rangle\right\rangle \Rightarrow\left\langle\left\langle x_{2}, x_{1}, l, t\right\rangle\right\rangle
$$

Proof: Since $\chi\left(x_{1}, x_{2}, d, t\right)=1$ and $d\left(x_{1}, x_{2}\right)=$ $d\left(x_{2}, x_{1}\right)$, we can derive $\chi\left(x_{2}, x_{1}, d, t\right)=1$.

For example, if we have 〈 Arisa, Bob, 0.5m, 2015-01$23 \mathrm{~T} 12: 34: 56\rangle\rangle$, then we also have $\langle\langle$ Bob, Arisa, 0.5m, 201501-23T12:34:56〉〉.

The next property is transitivity. When we have a pair of pass-by rendezvous, say " $A$ meets $B$ " and " $B$ meets $C$ ", simultaneously, we can infer " $A$ meets $C$ ".

Property P2 (Transitivity): Let $x_{1}, x_{2}$ and $x_{3}$ be mobile entities. Let $l_{1}=d\left(x_{1}, x_{2}\right), l_{2}=d\left(x_{2}, x_{3}\right), l_{3}=d\left(x_{1}, x_{3}\right)$. Let $L$ be the distance threshold. Let $t$ be a time-stamp. Then,

$$
\begin{aligned}
\left\langle\left\langle x_{1}, x_{2}, l_{1}, t\right\rangle\right\rangle & \wedge\left\langle\left\langle x_{2}, x_{3}, l_{2}, t\right\rangle\right\rangle \wedge\left(l_{1}+l_{2}<L\right) \\
& \Rightarrow\left\langle\left\langle x_{1}, x_{3}, l_{3}, t\right\rangle\right\rangle
\end{aligned}
$$

Proof: According to the triangle inequality, $d\left(x_{1}, x_{3}\right) \leq$ $d\left(x_{1}, x_{2}\right)+d\left(x_{2}, x_{3}\right)$. Since $l_{1}+l_{2}<L$, we have $l_{3} \leq l_{1}+l_{2}<$ $L$ at time $t$. Thus, we can derive $\chi\left(x_{1}, x_{3}, d, t\right)=1$.
For example, let $L$ be $1.0 \mathrm{~m}$. If we have 〈 $\langle$ Arisa, Bob, 0.4m, 2015-01-23T12:34:56 $\rangle$ and $\langle\langle$ Bob, Charley, 0.4m,

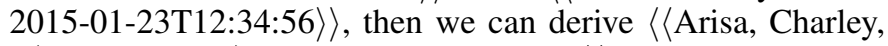
$d$ (Arisa,Charley), 2015-01-23T12:34:56 〉>

Note that $l_{1}+l_{2}<L$ is the sufficient condition for $\mathrm{P} 2$. Indeed, in some case $\left\langle\left\langle x_{1}, x_{3}, l_{3}, t\right\rangle\right\rangle$ holds even if $\left.l_{1}+l_{2}\right\rangle L$.

\section{Pass-by Rendezvous and Spatiotemporal Information}

The distance between any pair of mobile entities can be derived from the current location of the entities. This fact implies that a pass-by rendezvous can be inferred from known spatiotemporal information (i.e., data with location and time). Conversely, spatiotemporal information can be also estimated from known pass-by rendezvous.

In the following, we use notation $\langle x, p, t\rangle$ to represent a predicate that mobile entity $x$ is at position $p$ (with a location) at time $t$. Then, the following property holds.

Property P3 (Deriving pass-by rendezvous from spatiotemporal information): Let $x_{1}, x_{2}$ be mobile entities. Let $p_{1}, p_{2}$ be positions each of which has a location. Let $d^{\prime}$ be a distance function defined over the positions. Let $L$ be the distance threshold. Let $t$ be a time-stamp. Then,

$$
\begin{aligned}
\left\langle x_{1}, p_{1}, t\right\rangle & \wedge\left\langle x_{2}, p_{2}, t\right\rangle \wedge d^{\prime}\left(p_{1}, p_{2}\right)<L \\
& \Rightarrow\left\langle\left\langle x_{1}, x_{2}, d\left(x_{1}, x_{2}\right), t\right\rangle\right\rangle
\end{aligned}
$$

Proof: At time $t, x_{1}$ is at $p_{1}$, and $x_{2}$ is at $p_{2}$. So, $d\left(x_{1}, x_{2}\right)=d^{\prime}\left(p_{1}, p_{2}\right)<L$ at time $t$. Hence, we have $\chi\left(x_{1}, x_{2}, d, t\right)=1$.

Property P3 derives a pass-by rendezvous of $x_{1}$ and $x_{2}$ from the fact that their positions are sufficiently close. Even if $x_{1}$ or $x_{2}$ does not have a proximity sensor, the pass-by rendezvous can be inferred if individual locations are available.

The next property does the opposite, which estimates the spatiotemporal information from known pass-by rendezvous.

Property P4 (Estimating spatiotemporal information from pass-by rendezvous): Let $x_{1}, x_{2}$ be mobile entities. Let $p_{1}, p_{2}$ be positions each of which has a location. Let $d^{\prime}$ be a distance function defined over the positions. Let $L$ be the distance threshold. Let $t$ be a time-stamp. Then,

$$
\begin{aligned}
& \left\langle\left\langle x_{1}, x_{2}, l, t\right\rangle\right\rangle \wedge\left\langle x_{1}, p_{1}, t\right\rangle \\
& \quad \Rightarrow\left\langle x_{2}, p_{2}, t\right\rangle \text { for some } p_{2} \text { such that } d^{\prime}\left(p_{1}, p_{2}\right)=l
\end{aligned}
$$

Proof: At time $t, x_{1}$ and $x_{2}$ are at a distance of $l$, and $x_{1}$ is at $p_{1}$. Therefore, $x_{2}$ must be at a certain position $p_{2}$ whose distance from $p_{1}$ is $l$. So $\left\langle x_{2}, p_{2}, t\right\rangle$ and $d^{\prime}\left(p_{1}, p_{2}\right)=l$.

Property $\mathrm{P} 4$ estimates the position of $x_{2}$ from the given pass-by rendezvous of $x_{1}$ and $x_{2}$, and the position of $x_{1}$. Even if $x_{2}$ does not have a geolocation sensor, its position can be inferred from the pass-by rendezvous with located entity $x_{1}$.

\section{Effects of Device-Independent Pass-by Rendezvous}

The proposed definition provides a universal and abstract view of the pass-by rendezvous. This means that interoperability among heterogeneous pass-by systems can be achieved by 


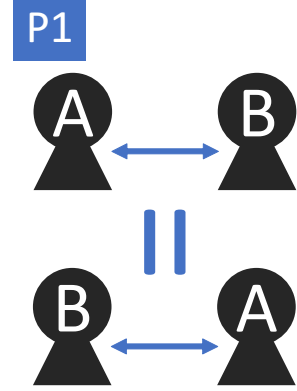

P2

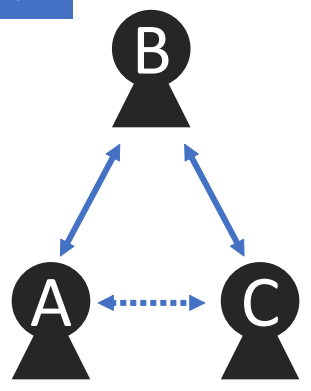

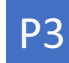

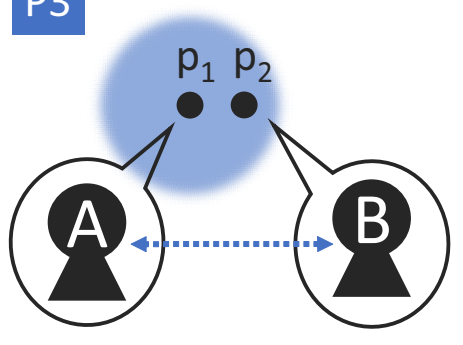

P4

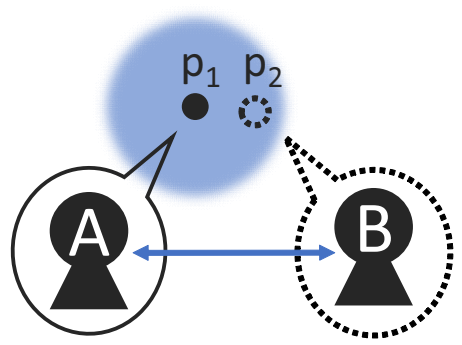

Fig. 2. Properties for Pass-by Rendezvous

adding an abstraction layer on top of the systems, based on the proposed definition.

The proposed properties can be fully utilized to derive indirect pass-by rendezvous. Figure 2 depicts intuitive illustration of Properties P1 to P4. In the figure, a fact derived from a property (which was unknown before) is drawn by a dotted line. Even if there is no direct way to detect a pass-by rendezvous of $x_{1}$ and $x_{2}$, the four properties may indirectly derive the rendezvous by connecting the existing facts.

By applying the four properties combinatorially to the existing records of pass-by rendezvous, we can generate a huge amount of data that detects many indirect pass-by rendezvous within larger areas. Note that the generated data is portable, which can be reused by various pass-by systems based on the device-independent definition.

Moreover, according to Properties P3 and P4, we can use spatiotemporal data for pass-by rendezvous, and vice versa. This implies that a pass-by system and a geolocation system (GPS, IPS, etc.) can be used together to complement mutual features and purposes. Thus, the proposed method is expected to expand the range of new applications and services with pass-by rendezvous.

\section{EXAMPLE APPLICATIONS}

This section describes the idea how to apply the proposed method to practical systems, taking BLE and GPS as examples.

\section{A. Bluetooth Low Energy (BLE)}

Bluetooth Low Energy (BLE) is a short-distance wireless communication technology that can be used for proximity sensing. The pass-by rendezvous can be implemented by using BLE with the GATT (Generic Attribute) profile. Each mobile entity is identified with proximity UUID and major/minor values. Mapping from these values to a user name (within the pass-by rendezvous world) is possible. The distance between two mobile entities can be estimated from RSSI (Received Signal Strength Indication) and Measured Power attributes.

Suppose that Arisa and Bob have smartphones with BLE for pass-by rendezvous. When Arisa passes by Bob, Arisa's smartphone receives a signal, decodes the name of Bob, and measure the distance. If the distance, say $0.7 \mathrm{~m}$, is below a predetermined threshold and it is 12:34:56 on 2015-01-23 now, then Arisa's smartphone emits a rendezvous event / Arisa, Bob, 0.7m, 2015-01-23T12:34:56 $\rangle$. If necessary, the event is saved to a $\log$ file or uploaded to a cloud server. The same thing can be done with Bob's smartphone, according to Property P1.

\section{B. GPS}

Gathering GPS information from individual mobile entities enables to detect pass-by rendezvous, as seen in Property P3. An approach is to deploy a cloud server and to make every mobile entity periodically send own ID and GPS data to the server. Every GPS data (usually represented in NMEA 0183 format) contains longitude and latitude, from which the distance between two points can be calculated.

Suppose that Bob and Charley are at location SIb and SIc, respectively, and that the current time is 12:34:56 on 201501-23. Bob (or Charley) sends an event Bob, SIb, 201501-23T12:34:56 (or 〈Charley, SIc, 2015-01-23T12:34:56〉, respectively) to the cloud server. On receiving the events, the server calculates the distance between SIb and SIc. If the distance, say $1.1 \mathrm{~m}$, is below a threshold, the server emits a ren-

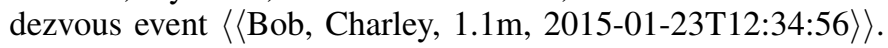

\section{Indirect Pass-by Rendezvous}

Suppose now that the distance threshold is $2.0 \mathrm{~m}$ and that we have the above two rendezvous / A Arisa, Bob,

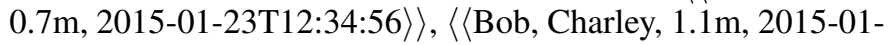
23T12:34:56 $\rangle$. Then, Property P3 derives 〈 1.8m, 2015-01-23T12:34:56 $\rangle$, which is an indirect pass-by rendezvous generated from the two different pass-by systems.

\section{ACKNOWLEDGMENT}

This research was partially supported by the Japan Ministry of Education, Science, Sports, and Culture [Grant-inAid for Scientific Research (C) (No.24500079, No.24500258), Scientific Research (B) (No.26280115), Young Scientists (B) (No.26730155)] and Kawanishi Memorial ShinMaywa Education Foundation.

\section{REFERENCES}

[1] S. Nomura, Y. Ho, E. Sato-Shimokawara, Y. Fujimoto, and T. Yamaguchi, "Information community system using pass-by wireless communication on smartphone," in Industrial Electronics (ISIE), 2013 IEEE International Symposium on, May 2013, pp. 1-6.

[2] T. Yamamoto and T. Tachibana, "Reliable data transmission with user's history for delay tolerant networks," in Technical report of IEICE, vol. 112, no. 209, 2012, pp. 93-98. [Online]. Available: http://ci.nii.ac.jp/naid/110009666456/

[3] “droidsensor," https://code.google.com/p/droidsensor/.

[4] Nintendo, "Nintendo 3DS - How StreetPass Works," https://www. nintendo.com/3ds/features/streetpass/how-it-works.

[5] Sony Computer Entertainment, "PS Vita - Near App," http://us. playstation.com/psvita/apps/psvita-app-near.html. 